This is an electronic reprint of the original article. This reprint may differ from the original in pagination and typographic detail.

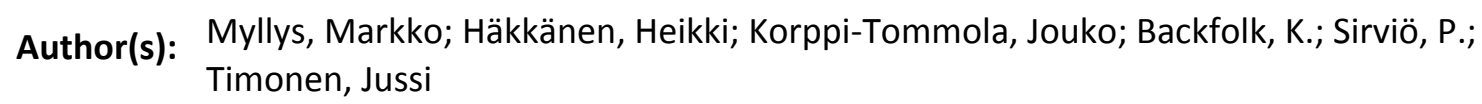

Title: X-ray microtomography and laser ablation in the analysis of ink distribution in coated paper

Year: $\quad 2015$

Version:

Please cite the original version:

Myllys, M., Häkkänen, H., Korppi-Tommola, J., Backfolk, K., Sirviö, P., \& Timonen, J. (2015). X-ray microtomography and laser ablation in the analysis of ink distribution in coated paper. Journal of Applied Physics, 117(14), Article 144902.

https://doi.org/10.1063/1.4916588

All material supplied via JYX is protected by copyright and other intellectual property rights, and duplication or sale of all or part of any of the repository collections is not permitted, except that material may be duplicated by you for your research use or educational purposes in electronic or print form. You must obtain permission for any other use. Electronic or print copies may not be offered, whether for sale or otherwise to anyone who is not an authorised user. 


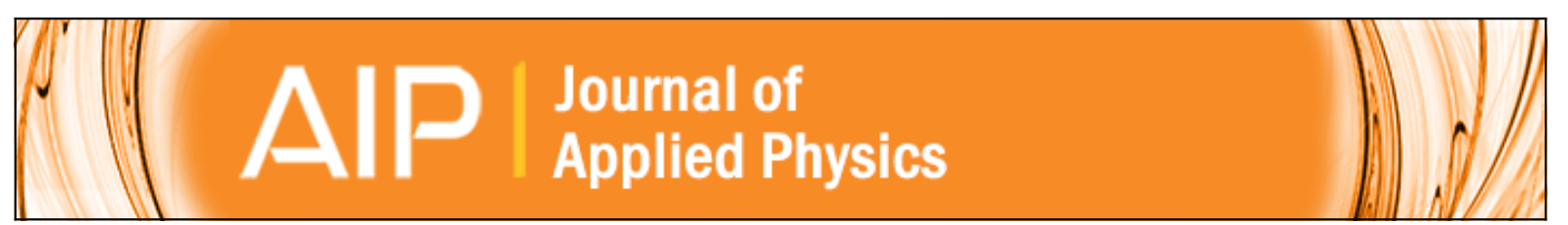

X-ray microtomography and laser ablation in the analysis of ink distribution in coated paper

M. Myllys, H. Häkkänen, J. Korppi-Tommola, K. Backfolk, P. Sirviö, and J. Timonen

Citation: Journal of Applied Physics 117, 144902 (2015); doi: 10.1063/1.4916588

View online: http://dx.doi.org/10.1063/1.4916588

View Table of Contents: http://scitation.aip.org/content/aip/journal/jap/117/14?ver=pdfcov

Published by the AIP Publishing

\section{Articles you may be interested in}

Multi-scale analysis in carbonates by X-ray microtomography: Characterization of the porosity and pore size distribution

AIP Conf. Proc. 1529, 86 (2013); 10.1063/1.4804091

Fast microtomography using bright monochromatic x-rays

Rev. Sci. Instrum. 83, 093704 (2012); 10.1063/1.4751853

Microstructure and deformation micromechanisms of concentrated fiber bundle suspensions: An analysis combining $\mathrm{x}$-ray microtomography and pull-out tests

J. Rheol. 56, 593 (2012); 10.1122/1.3698185

2D imaging by X-ray fluorescence microtomography

AIP Conf. Proc. 507, 539 (2000); 10.1063/1.1291207

Design of X-ray interferometer for phase-contrast X-ray microtomography

AIP Conf. Proc. 507, 98 (2000); 10.1063/1.1291125

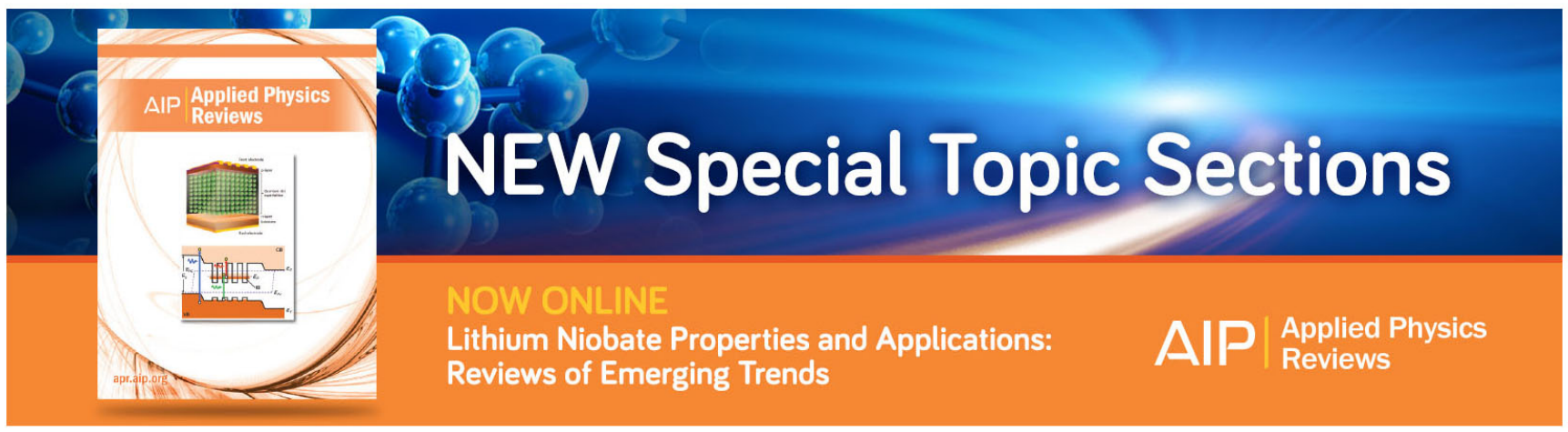




\title{
X-ray microtomography and laser ablation in the analysis of ink distribution in coated paper
}

\author{
M. Myllys, ${ }^{1, a)}$ H. Häkkänen, ${ }^{2}$ J. Korppi-Tommola, ${ }^{2}$ K. Backfolk,,${ }^{3,4}$ P. Sirviö, ${ }^{3}$ \\ and J. Timonen ${ }^{1,5}$ \\ ${ }^{1}$ Department of Physics and Nanoscience Center, University of Jyvaskyla, P.O. Box 35, FI-40014 Jyvaskyla, \\ Finland \\ ${ }^{2}$ Department of Chemistry and Nanoscience Center, University of Jyvaskyla, P.O. Box 35, FI-40014 Jyvaskyla, \\ Finland \\ ${ }^{3}$ Imatra Research Centre, Stora Enso Oyj, Tainionkoskentie 115, 55800 Imatra, Finland \\ ${ }^{4}$ Laboratory of Packaging Technology, Lappeenranta University of Technology, P.O. Box 20, \\ 53851 Lappeenranta, Finland \\ ${ }^{5}$ ITMO University, Kronverkskii ave. 49, 197101 Saint Petersburg, Russia
}

(Received 2 February 2015; accepted 20 March 2015; published online 14 April 2015)

\begin{abstract}
A novel method was developed for studying the ink-paper interface and the structural variations of a deposited layer of ink. Combining high-resolution x-ray tomography with laser ablation, the depth profile of ink (toner), i.e., its varying thickness, could be determined in a paper substrate. X-ray tomography was used to produce the 3D structure of paper with about $1 \mu \mathrm{m}$ spatial resolution. Laser ablation combined with optical imaging was used to produce the 3D structure of the printed layer of ink on top of that paper with about $70 \mathrm{~nm}$ depth resolution. Ablation depth was calibrated with an optical profilometer. It can be concluded that a toner layer on a light-weight-coated paper substrate was strongly perturbed by protruding fibers of the base paper. Such fibers together with the surface topography of the base paper seem to be the major factors that control the leveling of toner and its penetration into a thinly coated paper substrate. (C) 2015 AIP Publishing LLC.

[http://dx.doi.org/10.1063/1.4916588]
\end{abstract}

\section{INTRODUCTION}

Conveying information by printing text and images on various substrates (paper, plastics, card board, etc.) is a widely used technique with familiar applications in everyday life. Understanding the interplay between the structural properties of the substrate and the properties of the printing ink, and also those of other processes that affect the print quality, has, however, been a challenging task. In order to understand, e.g., the mechanisms by which the structural properties of the substrate influence the print quality, it is important to measure these properties with a very high spatial accuracy. Techniques such as, e.g., scanning electron microscopy (SEM) would provide the necessary accuracy, but the spatial information would be restricted to two dimensions (2D). A combination of 2D SEM images into a 3D stack would be a very slow and demanding task.

The spatial accuracy of $\mathrm{x}$-ray tomography has improved dramatically during the last few years, and a sub-micrometer resolution has become possible even in the laboratory. This improvement has opened up new possibilities in the 3D structural analysis of numerous inhomogeneous materials such as, e.g., paper. A 1- $\mu$ m resolution makes it possible, for example, to do a rather detailed analysis of coated papers used as printing substrates. A non-destructive method like $\mathrm{x}$-ray tomography will provide new and fascinating insight into the spatial variation of rough composite substrates that display both micrometer-level and sub-micron porosity.

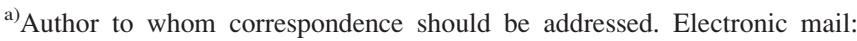
markko.myllys@jyu.fi
}

The characteristics of the ink-substrate interface are crucial for the final properties of print, such as, e.g., its visual appearance and durability. In electrophotography, for instance, there is an increasing trend toward use of engineered toner particles that allow different physical and chemical modifications. The aim of this development is to improve the actual printing process, like the runnability of the printing machine, but also the print quality, and to lower the production and print costs. So as to be successful, such developments require a particularly good understanding of the ink-paper interface, since the amount of ink will be minimized in the deposited layer of ink.

As already discussed above, SEM imaging is frequently used to analyze ink on a substrate. This method cannot provide, as discussed, 3D information about the ink-substrate behavior. Hakola et al., for instance, ${ }^{1}$ have used model substrates to demonstrate with SEM that toner penetration is limited, when the pore size approaches $100 \mathrm{~nm}$ in diameter. Vikman and $\mathrm{Sipi}^{2}$ have used FTIR and Raman to get information about the characteristics of a toner-paper interface, but they have also shown that the varying absorbance by different toner components of the optical signal may influence the interpretation of the results. Iwata et $a l^{3}{ }^{3}$ have made a chemical mapping of the toner components using a scanning $\mathrm{x}$-ray method, and have demonstrated that a highresolution imaging of toner penetration is possible although challenging by this method. Spectroscopic imaging would also be challenging since lateral variations of the toner composition influence the resolution of the created 3D image. The error in the toner would become even more 


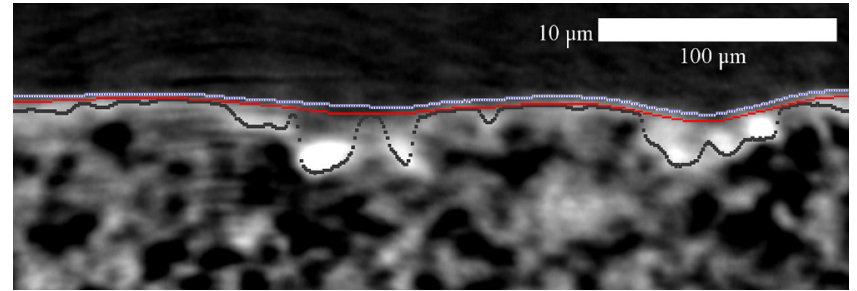

FIG. 1. A cross-section of a toner layer (between the dashed blue and continuous red lines) and a coating layer (between the continuous red and thick grey lines) above the base paper, as determined from a tomographic reconstruction of a sample by the qEW method.

severe if the toner composition would be complex and unknown.

Most of the methods described above can provide only limited information about the role of substrate properties in the distribution of ink. Some recent works have already demonstrated the benefits of using synchrotron or x-ray tomography in understanding the toner-substrate interaction. ${ }^{4-6}$ Combining three-dimensional tomographic data and surface topography, the local optical properties of printed paper have been analyzed. ${ }^{7}$ It is, however, crucial to be able to determine the base-paper structure and the toner-layer characteristics at the same location since the distribution of ink and the substrate structure both vary appreciably. Often their deviations from their average values are more significant than the mean values themselves.

In the present work, the material heterogeneity of the substrate, i.e., paper with a thin coating layer, was determined by $x$-ray tomography with about $1-\mu$ m resolution. The isotropic resolution of the $\mathrm{x}$-ray tomography was good enough to resolve the $3 \mathrm{D}$ distribution of the coating layer and its correlation with the both surface topography and structure of the base paper. This resolution was quite adequate in the lateral dimensions, but a clearly better resolution in the vertical direction was needed, since the ink layer was very thin. To this end, we used laser ablation combined with optical imaging, so as to be able to analyze the ink distribution above the known micrometer-scale structure. In this way, we could obtain about 50-nm resolution in the vertical direction, while the lateral resolution was set by that (a nominal resolution of $0.8 \mu \mathrm{m}$ ) of the device set-up. ${ }^{8}$ The ablation depth could be changed continuously by changing the intensity of the laser pulse, and lateral toner distributions in each ablation layer could be easily detected by optical imaging. Since the true ablation depths created by application of the laser pulses depended on the local material density, the depths of ablation "craters" were also calibrated by the same optical profilometer.

The x-ray tomography and ablation images were then combined so as to obtain a realistic 3D image of the toner distribution on a coated paper with a known micrometerscale structure. For the substrate, we designed a rather thinly coated (coat weight $5 \mathrm{~g} / \mathrm{m}^{2}$ ) paper, which displayed a significant variation in its surface structure, and demonstrated thereby the strength of the methodology used. The results obtained allowed us to discuss the implications of an uneven porosity and the pore-size distribution on the leveling of toner and its distribution in the substrate.

\section{MATERIALS AND METHODS}

A $90 \mathrm{~g} / \mathrm{m}^{2}$ base paper was made on a pilot machine, and then coated with a bench-rod coater. The targeted weight of the coating layer per side was $5 \mathrm{~g} / \mathrm{m}^{2}$. The composition of the coating was 100 parts of calcium carbonate and 10 parts of styrene/butadiene latex, and 1 part of sodium-carboxymethyl cellulose. The wood-free base paper contained 15\% PCC filler, and was calendered before coating. The coated base paper was in fact calendered twice in the laboratory with a soft nip, at a nip pressure of $25 \mathrm{kN}$ and at a temperature of $100^{\circ} \mathrm{C}$. The coated paper was printed in an electrophotographic network printer that used a chemical toner based on styrene/ acrylate chemistry.

The x-ray microtomography $(\mu \mathrm{CT})$ can provide the internal (3D) mass distribution of the sample based on the measured attenuation of x-rays. For 3D imaging, attenuation was measured in a number of different directions. The structure of the sample was then reconstructed from the recorded 2D attenuation images using an inversion algorithm. A Skyscan 1172 system with a voxel size of $0.8 \mu \mathrm{m}$ was used for imaging. ${ }^{7}$

Surface topographies of the both toner and coated paper were segmented from the reconstructed 3D image using a surface-detection algorithm based on the quenched EdwardsWilkinson (qEW) equation. ${ }^{9}$ This technique is a slightly simplified version of the so-called snakes model, ${ }^{10}$ especially designed for analysis of 3D tomographic images. In this technique, a 2D surface, whose dynamics is governed by the qEW equation, approaches the interface to be segmented in such a way that the gray-scale values of the image exert forces that oppose the motion of the surface. By tuning the
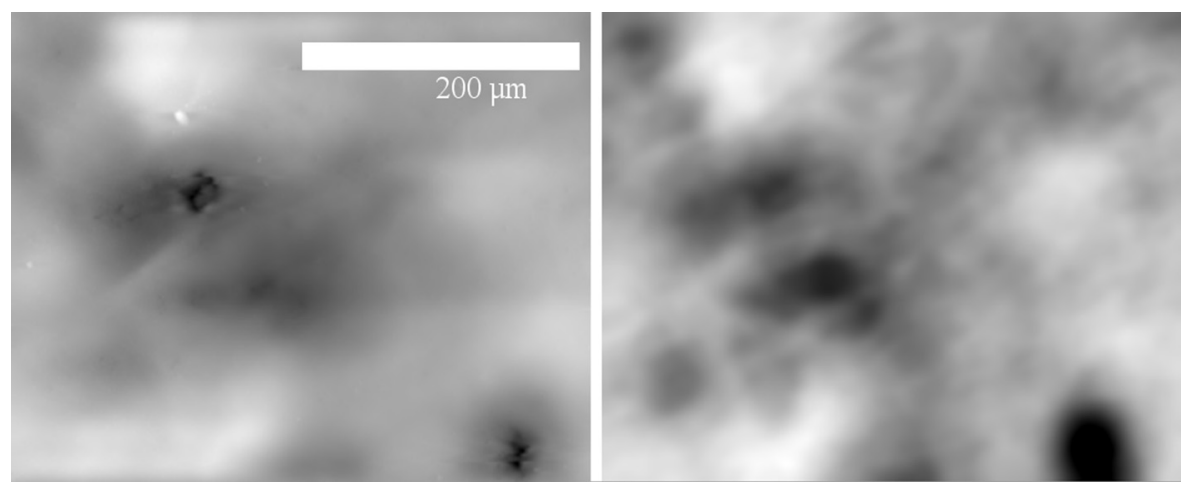

FIG. 2. Topographic maps in gray scale of the same $418 \times 338 \mu \mathrm{m}^{2}$ area of the substrate as determined by surface profilometry (left panel) and x-ray tomography (right panel). 


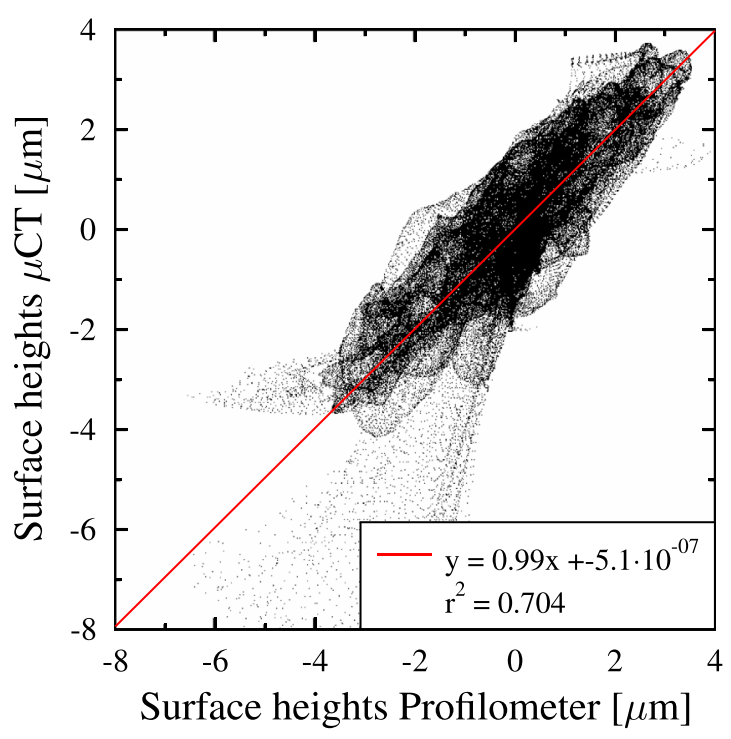

FIG. 3. Height-height cross-correlation data of the two images of Fig. 2 together with a linear fit (red solid line) to the data. Notice that the outlier points do not affect much this correlation as their density is low, and thus they were not analyzed further.

parameters of the technique, the surface stops exactly at the interface without much effect from the ever-present noise. By re-tuning the parameters, a stopped surface will continue its advance, and will stop at the (spatially) next interface if it exists. Because of using this technique, no other imageanalysis techniques and noise-reduction methods were needed in the analysis. The $\mathrm{qEW}$ method is so accurate that we could detect details of the sample at fairly near the pixel resolution of the tomographic image. As an example, we show in Fig. 1 segmented layers of toner and coating. In the results reported below, for the distribution of toner thickness we, however, used the laser-ablation result that was even more accurate.

An ArF excimer laser (OPTex, Lambda physik $\mathrm{GmbH}$ ) was used to ablate toner from prints on paper substrates. ${ }^{8,11}$ This laser was operated with a fluence of $0.07 \mathrm{~J} / \mathrm{cm}^{2}$ and a wavelength of $193 \mathrm{~nm}$. In order to obtain a flat-top beam profile, an aperture of $3 \mathrm{~mm}$ in diameter was used, to allow only the center part of the laser beam to reach the sample. The focus of the laser beam was positioned in front of the sample surface. This arrangement gave a more uniform bottom for the ablation craters than focusing the beam inside the sample. The diameter of the laser spots was adjusted to $0.6 \mathrm{~mm}$ by fixing the lens-to-sample distance, and the energy of the laser pulses was set to $0.4 \mathrm{~mJ}$. The spot area was determined by imaging the ablation craters with a digital camera (Canon Inc.) connected to an optical microscope. The depths of these craters were calibrated by optical profilometry (the surface profiles of selected samples were obtained using a Rodenstock RM-600 white-light laser profilometer operated at $5 \mathrm{~mm}$ lines, using $2.5-\mathrm{mm}$ steps, and with a depth resolution less than $1 \mu \mathrm{m})$. The mean ablation rate for the used fluence, $70 \mathrm{~nm} /$ pulse, was obtained by dividing the total depth of the ablation crater by the number of laser pulses used to create it. Images of the ablation craters were stored on a computer for a later analysis. The toner thickness was analyzed in areas of complete coverage by a cyan toner. The criterion for having the toner in the optical images was chosen such that the ratio of red and green false-color intensities in them was less than 0.65 .

\section{RESULTS AND DISCUSSION}

Each paper sample of $(1 \times 1) \mathrm{mm}^{2}$ was scanned by the $\mathrm{x}$-ray tomography device and the surface profilometer (Fig. 2), and imaged with a conventional optical microscope, both before and after the ablation (Fig. 3). An area outside the ablation craters in the microscope image was used to mask the image taken before ablation.

We first tested how accurately the surface topography of the samples was produced by $\mathrm{x}$-ray tomography in comparison with the surface profilometry in which the vertical resolution was much higher. We show in Fig. 2 topographic maps taken from the same area by these two methods.

It is evident from Fig. 2 that profilometry and tomography results for the surface topography were very similar. So as to quantify this similarity, we calculated the cross correlation of the two gray-scale images. The resulted crosscorrelation plot is shown in Fig. 3.

Linear regression of the cross-correlation plot gave $\mathrm{R}^{2}=0.70$ for the correlation coefficient, which means that correlation was good. The spatially varying thickness of the toner layer was measured by laser ablation combined with optical imaging as described above. A thickness map of the
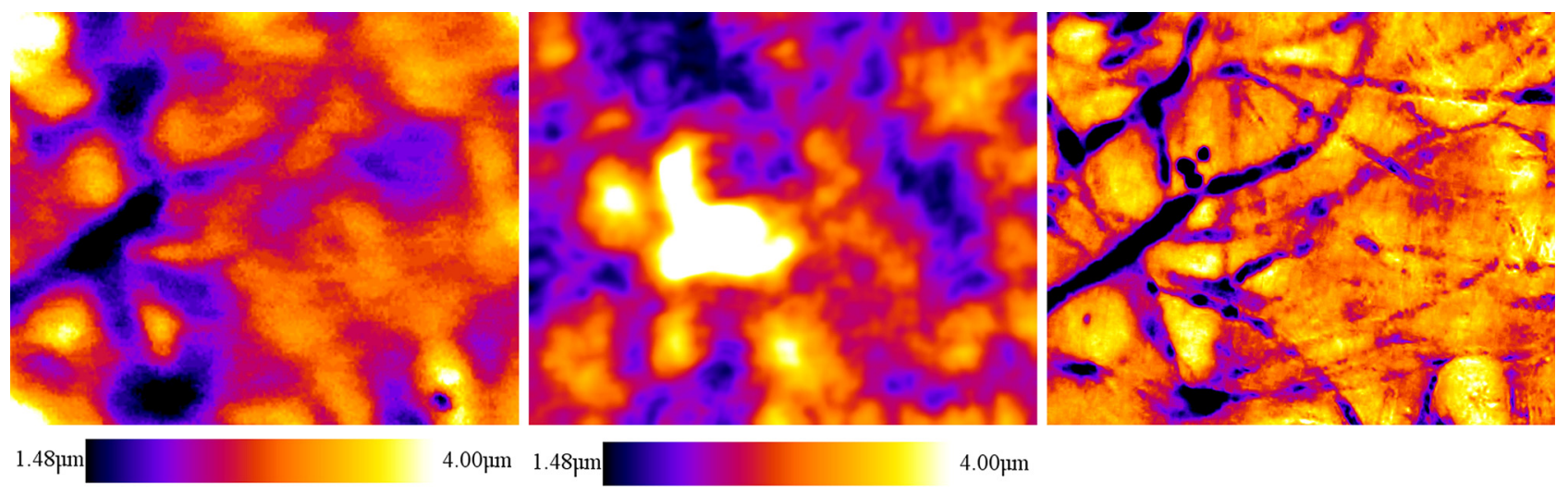

FIG. 4. The thickness map of the toner as determined by laser ablation (left), x-ray tomography (middle), and intensity of through-transmitted red light (right). The darker the color, the less there is toner. 


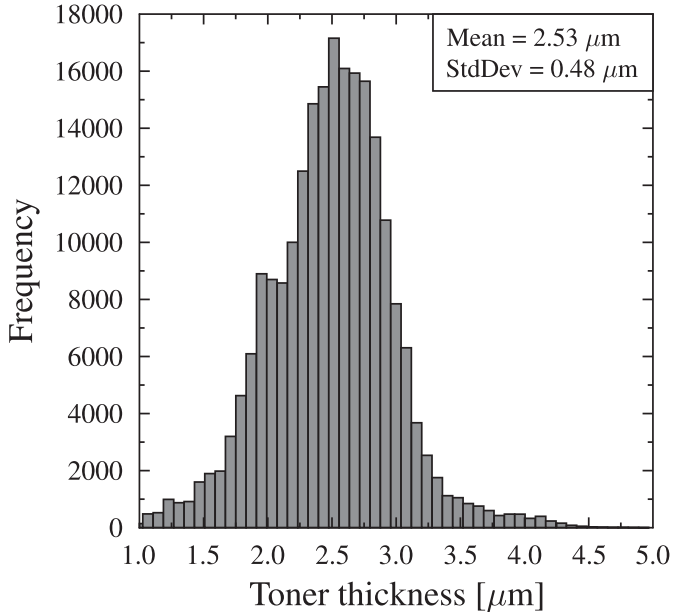

FIG. 5. Distribution of the toner thickness $(\mu \mathrm{m})$ in the laser-ablation map (left panel) of Fig. 4.

toner is shown in Fig. 4 (left). In the same figure (right), we also show the intensity of the red color of an optical image with through illumination of the same spot, taken before the ablation. We also tried to extract the toner layer from the tomographic images with the qEW algorithm, but because of limited resolution the accuracy of the extracted layer was not good enough for a quantitative analysis. However, it is evident from the segmented tomographic images that the thickness of the toner layer varied appreciably and was strongly dependent on the roughness of the substrate. In the areas where protruding fibers existed on the surface of the base paper, the toner layer was thinner (fusing smoothens the toner layer by transferring toner into the cavities of the coating).

It is evident from the thickness distribution of the leftmost panel of Fig. 4, shown in Fig. 5, that, although it is clearly peaked, it is fairly broad. There were no areas of zero toner thickness in Fig. 4 (left), and the average thickness was about $2.5 \mu \mathrm{m}$, which is to be expected for the chemical toners used in modern electrophotographic printers.

The toner thickness appears thus to be related to the presence of wood fibers and coating pigments in the surface layer of the base paper, and it correlates with the transmission of red light through the paper. Cyan color of the toner absorbs effectively red light, so correlation is strongest for

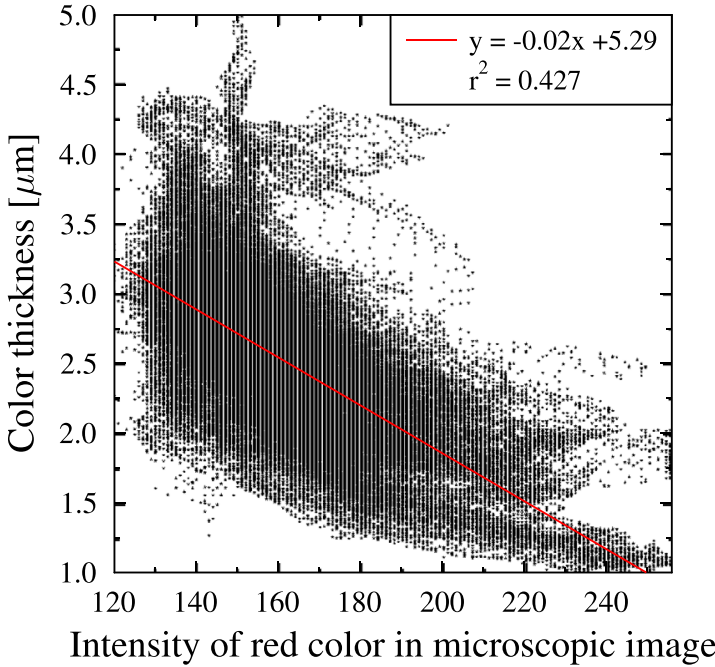

FIG. 6. Correlation between the toner thickness, as determined by laser ablation, and the intensity of red light transmitted through the sample. Notice that no attempt was made to remove the statistical outliers.

this color. There is a clear correlation between the toner thickness and the transmitted light intensity (Fig. 6).

The local surface elevation was correlated, albeit rather weakly, with the local toner thickness: There was a tendency that areas with a toner thickness below the average were located at higher elevations and vice versa (see Fig. 7 (left)). Best two-point correlation of toner thickness was found, when the surface topography was coarse-grained into $30 \mu \mathrm{m}$ accuracy, using FFT and band-pass filtering. Also, other topological features of the surface, such as the magnitude and orientation of its first and second derivatives were determined. ${ }^{12}$ The toner thickness correlated best with the second derivative of the surface, when determined in a $50 \mu \mathrm{m}$ length scale (see Fig. 7 (right)).

One would expect the effect of coating to be more important. For the $5 \mathrm{~g} / \mathrm{m}^{2}$ coat weight that was applied, the coating covered about $30 \%$ of the surface of the base paper, which is very much as expected. This substrate could thus rather be classified as a surface-hole-filled paper than a coated paper.

It should be noted that the average surface elevation was lower in coated than in uncoated areas. This is due to a very thin coating in blade-coated samples: much of the coating was
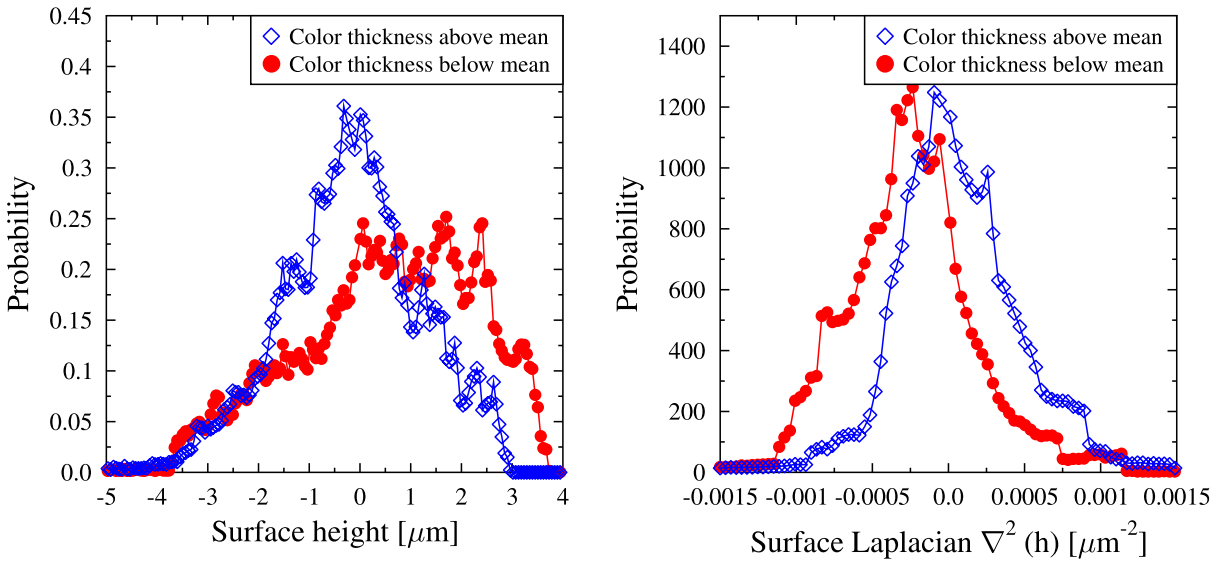

FIG. 7. Probability distributions of the above-average (blue diamonds) and below-average (red circles) toner thicknesses as a function of surface height with respect to the average height (left panel) and as a function of the second derivative of the surface (right panel). Second derivative was determined for a surface that had been coarse-grained to a $50 \mu \mathrm{m}$ resolution. 


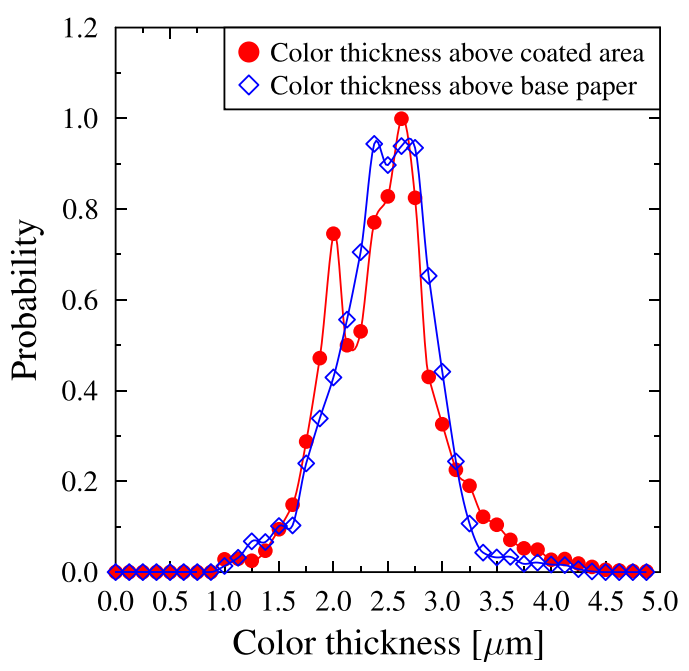

FIG. 8. Distribution of the toner thickness as determined by laser ablation for coated (red circles) and uncoated (blue diamonds) parts of the substrate.

located in depressions of the paper surface, and even after application of the coating, the surface height of the coated areas remained below the average height of the surface. Fibers that protruded out of the surface of the base paper had a thinner layer of toner, as is already obvious from Figs. 4, 8, and 9, and toner did not fill the depressions of uncoated areas as they were rather deep. On the coating, the toner layer was thus rather homogeneous, and depressions filled with coating had become relatively shallow. This is the origin of the correlation observed between the toner thickness and the surface elevation.

We can finally visualize the toner-substrate system combining the image of the toner layer as determined by laser ablation with that of the substrate as determined by x-ray tomography. Such a construction is shown in Fig. 9. It is evident from this figure that the toner thickness is not dominated by the surface structure of the substrate alone. As reported by, e.g., Mitsya, ${ }^{13}$ Petterson, ${ }^{14}$ and Backfolk et al. ${ }^{15}$ deposited toner particles merge and coalesce so as to form an even surface, especially at higher fusing temperatures that appear in the radiation curing or at longer nip-fusing times. It was also suggested there that not all the irregularities in the toner surface, as detected by surface profilometry, can be observed in its gloss data, but, depending on the band of light used, and on the type of color and amount of ink, different correlations will be seen. Although Pettersson ${ }^{14}$ used a radiation fuser, and a different ink and ink-transfer system, it is obvious from the present results that the surface profile and ink thickness do not necessarily follow closely the substrate topography,

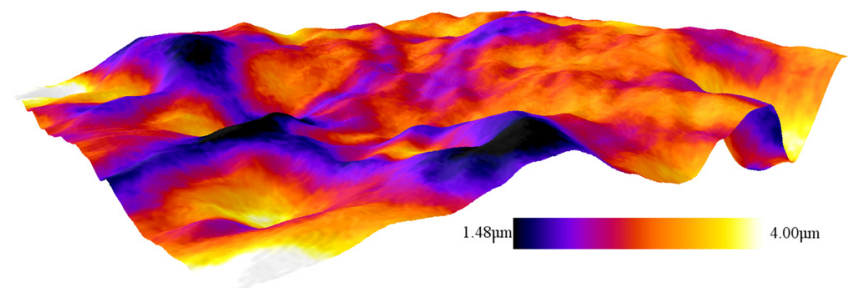

FIG. 9. Three-dimensional visualization of the toner layer, as determined by laser ablation, on top of a substrate of coated paper, as determined by x-ray tomographic reconstruction. Blue color denotes the (thin) toner layer. which suggests that there is very little correlation between the surface texture and, e.g., the print gloss in agreement with the results of Pettersson ${ }^{14}$ or those of Sirviö and Backfolk. ${ }^{16}$ As the toner transfer depends quite much on the texture and electrical properties of the surface, ${ }^{15,16}$ and the subsequent toner fusing depends on the packing properties of the toner, ${ }^{13}$ the resulting thickness of the coalesced and fused ink layer may display significant variations. Nevertheless, the method based on combining $\mathrm{x}$-ray tomography with laser ablation proved to be very powerful in the analysis of ink thickness and its local variations, and in demonstrating that the transfer and leveling of the toner onto a composite substrate do not show materialcomponent-dependent heterogeneities. This method would further benefit from combining it with spectroscopic methods, so as to analyze the distribution of chemical components at the substrate surface, and their possible impact on the adhesion of a toner. ${ }^{2}$

\section{CONCLUSIONS}

We demonstrated that, by combining laser ablation of a very-high vertical resolution with $\mathrm{x}$-ray tomography of a resolution of about a micrometer, it is possible to analyze in detail the distribution of toner on a substrate such as coated paper. Comparing the results obtained by this combination of methods with those of more conventional profilometry indicated that the present topographic map of sample surface provided a more reliable representation of surface structures. Combining the distribution of the toner thickness, as determined by laser ablation, with the surface structure, as determined by tomography, produced a very realistic picture of the interplay between the toner and the substrate.

We used paper with a low coat weight in order to demonstrate the impact of heterogeneous substrate composition on the toner distribution and subsequent print quality. The toner thickness appears to be rather dictated by the local roughness variations of the substrate surface than the chemical heterogeneity that arises from its partial fiber coverage. Wood fibers protruding out of the base-paper surface were relatively thinly covered by the toner, and showed up clearly in its thickness map, contributing thus to the negative correlation between the toner thickness and surface elevation. Because of these features, transmission of intensive red light effectively absorbed by the cyan color of the toner could also be used to determine the gross thickness variations of the toner, at least on this thinly coated base paper.

We believe that the methods developed in this work can be effectively used to analyze in detail the mechanisms that affect the print quality, which are of great practical importance, but have been difficult to analyze until now.

\section{ACKNOWLEDGMENTS}

The Finnish Government Centre for Advancement of Technology (TEKES) is gratefully acknowledged for financial support Project No. 3113/31/04.

${ }^{1}$ E. Hakola, J. Koskinen, U. Forsström, and P. Oittinen, “Toner penetration into porous substrates," J. Imaging Sci. Technol. 52(4), 040501-1-040501-8 (2008). 
${ }^{2}$ K. Vikman and K. Sipi, "Applicability of FTIR and Raman spectroscopic methods to the study of paper-ink interactions in digital prints," J. Imaging Sci. Technol. 47(2), 139-148 (2003).

${ }^{3}$ N. Iwata, A. Watada, K. Tani, H. Ikeura-Sekiguchi, T. Araki, and A. P. Hitchcock, "Application of scanning transmission X-ray microscopy for observation of organic compounds in toner particles," in International Conference on Digital Printing Technologies (2007), pp. 248-251.

${ }^{4}$ J.-F. Bloch, S. Rolland Du Roscoat, C. Mercier, P. Verhnes, B. Pineaux, A. Blayo, and P. Mangin, "Influence of paper structure on printability: Characterisation of paper using X-Ray synchrotron microtomography," in International Conference on Digital Printing Technologies (2006), pp. 449-453.

${ }^{5}$ P. Vernhes, S. R. Du Roscoat, A. Blayo, B. Pineaux, X. Thibault, and J.-F. Bloch, "Synchrotron X-ray microtomography: A new tool to characterize the interaction between paper and toner," J. Imaging Sci. Technol. 52(1), 010502-1-010502-6 (2008).

${ }^{6} \mathrm{G}$. Chinga-Carrasco, "Exploring the multi-scale structure of printing paper-A review of modern technology," J. Microsc. 234(3), 211-242 (2009).

${ }^{7}$ M. Juuti, T. Prykäri, E. Alarousu, H. Koivula, M. Myllys, A. Lähteelä, M. Toivakka, J. Timonen, R. Myllylä, and K.-E. Peiponen, "Detection of local specular gloss and surface roughness from black prints," Colloids Surf., A 299(1-3), 101-108 (2007).

${ }^{8}$ H. Häkkänen, J. Houni, S. Kaski, and J. E. I. Korppi-Tommola, "Analysis of paper by laser-induced plasma spectroscopy," Spectrochim. Acta, Part B 56(6), 737-742 (2001).
${ }^{9}$ G. Chinga-Carrasco, H. Kauko, M. Myllys, J. Timonen, B. Wang, M. Zhou, and J. O. Fossum, "New advances in the 3D characterization of mineral coating layers on paper," J. Microsc. 232(2), 212-224 (2008); T. Turpeinen, M. Myllys, P. Kekäläinen, and J. Timonen, "Interface Detection Using a Quenched-noise Version of the Edwards-Wilkinson Equation," IEEE Transactions on Image (submitted).

${ }^{10}$ M. Kass, A. Witkin, and D. Terzopoulos, "Snakes: Active contour models,” Int. J. Comput. Vision 1(4), 321-331 (1988).

${ }^{11}$ J. Kansanaho, J. E. I. Korppi-Tommola, and H. Hakkanen, "Print quality vs. coat weight and binder distribution in coated paper," in TAPPI Advanced Coating Fundamentals Symposium (2001), pp. 47-52.

${ }^{12}$ G. Chinga, P. O. Johnsen, R. Dougherty, E. L. Berli, and J. Walter, "Quantification of the 3D microstructure of SC surfaces," J. Microsc. 227(3), 254-265 (2007).

${ }^{13}$ T. Mitsuya and M. L. Hunt, "Toner particle packing in an electrostatic field and its effect on heating in electrophotography," Powder Technol. 92(2), 119-125 (1997).

${ }^{14} \mathrm{~T}$. Petterson, "Wetting and levelling of toner during fusing of electrophotographic prints," Licentiate thesis (KTH, Sweden, 2004).

${ }^{15}$ K. Backfolk, P. Sirviö, P. Ihalainen, and J. Peltonen, "Thermal and topographical characterization of polyester- and styrene/acrylate-based composite powders by scanning probe microscopy," Thermochim. Acta 470(1-2), 27-35 (2008).

${ }^{16} \mathrm{P}$. Sirviö and K. Backfolk, "Effect of roughness of low-grammage coated papers on print quality in color electrophotography," J. Imaging Sci. Technol. 52(1), 010505-1-010505-9 (2008). 